\title{
The simulation of operating of a positioning system actuated by electrical stepping motor
}

\author{
Dana Rizescu ${ }^{1}$, and Ciprian Ion Rizescu, ${ }^{1, *}$ \\ ${ }^{1}$ University POLITEHNICA of Bucharest, Mechatronics and Precision Engineering Department, 313 \\ Splaiul Independentei, 060042, Bucharest, Romania
}

\begin{abstract}
The work presents the current situation concerning positioning systems manufactured by well-known companies in the industry: Festo, SMC, etc, or patented solutions for positioning system. The authors suggest, following a thorough analysis, as a original solution, a positioning system driven by an electrical stepping motor. There are presented some particular elements of design, modeling, execution, testing and optimization of the proposed system. For validation, the mathematical model, authors have developed, in Matlab environment, a graphic user interface in which one can simulate the operation of several solutions with stepping motors, different positioning strokes.
\end{abstract}

\section{Introduction}

There is known that among the benefits of the pneumatic systems may be: rapid operation, easy maintenance, flexibility in use, good weight /power ratio, are clean, properties of selfcooling and the relatively low level costs. In comparison with hydraulic actuators, in the case of pneumatic actuators there is no risk of contamination of the driven load with leaking liquids and are not necessary additional mechanisms that transforms the rotation in linear movement such as the cases in which use electric rotational motors. Another important advantage is the availability of compressed air in most industrial units. All of these features have been immeditately tappet in a large number of applications in the fields of robotics, machine tools, machines, the processing industry, food processing systems, in the healthcare and rehabilitation systems.

There are known solutions of some companies in the field the precise adjustment of the position such as: Festo, SMC but we have to mention here the solutions of patents. The authors have taken into account more solutions. One of them is to be known from the U.S. Patent 6,802,242 B1, and shall relate to the control of the flow of fluid and in particular a control system for an air circuit with improvement in the sensitivity of the cylinder carried out through the use of selective control valve for the components of the sealing $\mathrm{O}$ ring at each end of the cylinder [1]. In such applications, it may be desirable a quick operation and in a repeated positioning of the piston with the precision of a thousandths of an inch.

\footnotetext{
* Corresponding author: ciprian.rizescu@,upb.ro
} 
Another solution is that of the US 7,021,191 B2 patent, where is described a position control system that is used to control a pneumatic cylinder having a room, defined by a piston, located in a housing for moving between two proportional valves for the flow control, electrically actuated and connected to each union of the cylinder, to control the flow of fluid selectively and proportional in one of the chambers [2]. A pressure sensor is provided for measuring the pressure of the fluid corresponding to each rooms.

\section{Experimental Setup}

In figure 1 is presented the principle sketch of the solution proposed, in which there were noted: M - guided mass which must be stopped with high precision, PLM - pneumatic linear motor, UPO - stop unit with high precision, S1, S2 - sense valves, DP - pneumatic distributors, EM - electromagnets, $\mathrm{O}$ - stopper, $\mathrm{SP}$ - position sensor.

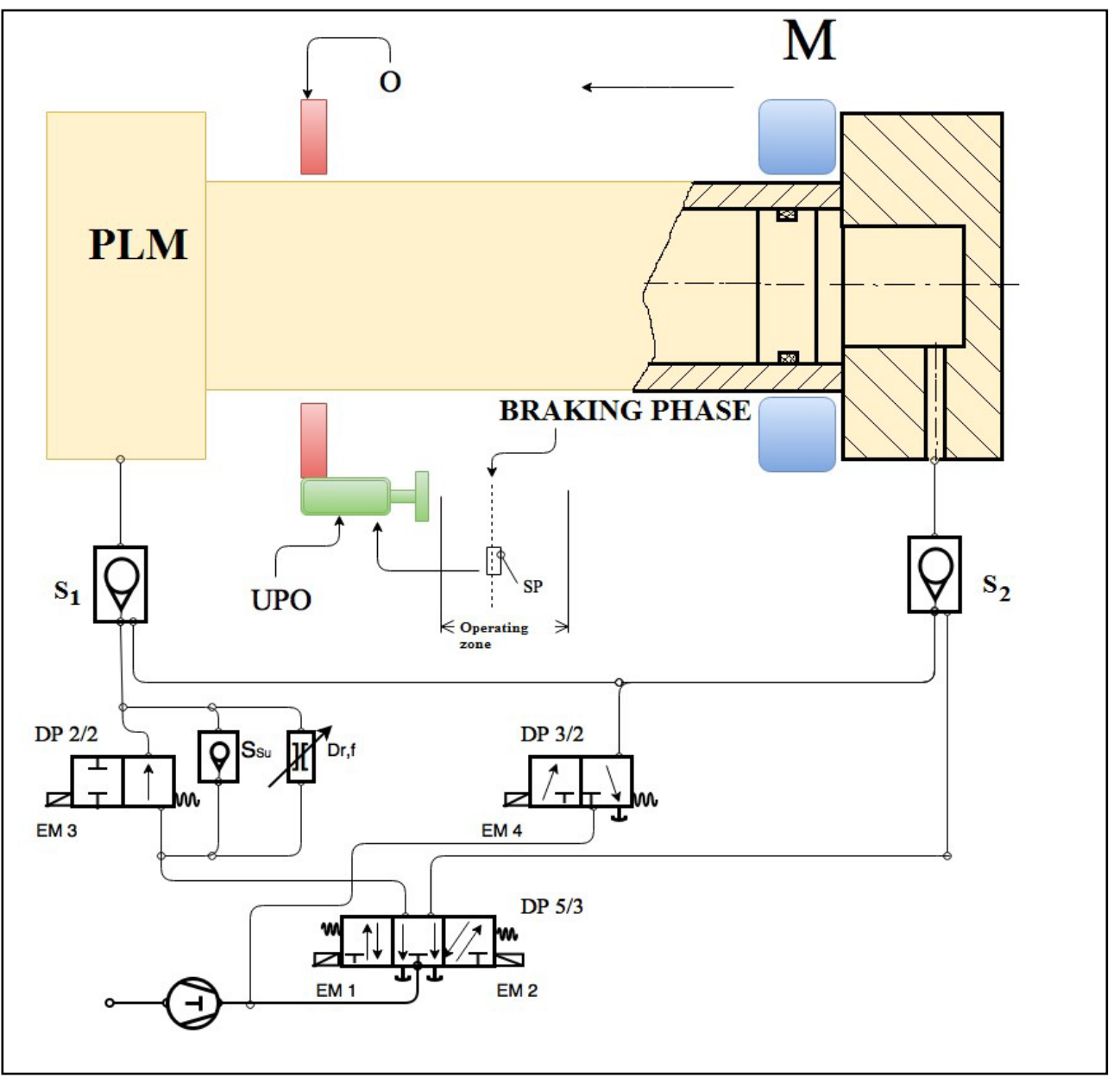

Fig. 1. Principle sketch of the experimental setup.

The operation mode of the equipment. The incremental electric motor (stepping motor) operate for positioning the stopper $\mathrm{O}$ in the desired position and locks the stopper to prevent the displacement when the contact with the mobile assembly is obtained [3]. The stepping motor actuates a nut-screw mechanism for a high precision positioning. Then switch the 
pneumatic distributor DP1 toward the right, feeding with air the room from the right-hand side of cylinder. The cylinder piston moves to the left. When the mobile assembly shall enter into range of the position sensor, SP, this command the pneumatic distributor DP2 which closes. At this time starts the braking process of piston (mobile assembly) as follows: air is purged from the camera from the left hand side of the cylinder through restrictor Dr, achieving an air cushion (therefore a back-pressure) in left hand side of the cylinder. The cylinder piston continue driving on at low speed. Selective unlockable sense valves S1 and S2 air locks cylinder rooms, this being stopped firmly on position.

The authors have developed an graphic user interface in the Matlab environment for simulating the stop unit operation, considering the various electric stepping motors having different step angles. As a result, one can study different actuation solutions for the stepper motors with the different step angles.

\section{Experimental Setup Simulation}

There is known that there are three commonly used excitation modes for step motors; these are full step, half step and microstepping.First mode: in full step operation, the motor moves through its basic step angle, i.e., a $1.8^{\circ}$ step motor takes 200 steps per motor revolution. There are two types of full step excitation modes. In single phase mode, also known as "one-phase on, full step" excitation, the motor is operated with only one phase (group of windings) energized at a time [4]. This mode requires the least amount of power from the driver of any of the excitation modes. In dual phase mode, also known as "twophase on, full step" excitation, the motor is operated with both phases energized at the same time. This mode provides improved torque and speed performance. The second mode: half step excitation is alternating single and dual phase operation resulting in steps that are half the basic step angle. Due to the smaller step angle, this mode provides twice the resolution and smoother operation. Half stepping produces roughly near $15 \%$ less torque than dual phase full stepping. The third mode: microstepping is a technique that increases motor resolution by controlling both the direction and amplitude of current flow in each winding. Current is proportioned in the windings according to sine and cosine functions. Microstepping can divide a motor's basic step up to 256 times. Microstepping improves low speed smoothness and minimizes low speed resonance effects. Microstepping produces roughly $30 \%$ less torque than dual phase full stepping.

The graphic user interface - main panel is presented in figure 2. There are some fields that must be filled in or some pop-up selection have to be made. The cylinder stroke range runs from $100 \mathrm{~mm}$ to $350 \mathrm{~mm}$ [5-6]. This stroke represents the displacement of guided mass M (figure1). For example, the cylinder stroke was considered $225 \mathrm{~mm}$, then there was selected motor 1 from a database developed by authors. There were considered some representative stepping motors with different number of windings, step angles and excitation modes [7]. In figure 2 was selected motor 1 with Torque diagram shown in a dedicated window. When this selection is completed a motor characteristic is displayed [8]. The motor excitation mode can be chosen : stepping or microstepping. The authors used for this exeprimental setup full step and half step excitation modes. The stepping motor stroke could be chosen up to $20 \mathrm{~mm}$. This stroke represents the displacement of the UPO unit, actuated with the stepping motor. If the operator "forget" to fill in one field or to choose one selelction a error message will be displayed on the screen. At this moment the SIMULATION button has to be pressed.

In the first step, the stepping motor stroke is set, considering the actuation mode, the desired stroke. This simulation is shown in figure 3. The number of steps/10 versus stepping motor stroke is presented also in the figure 3. After the motor stroke is set the 
system is ready to actuate the slider mass $\mathrm{M}$. There is another window which presents the impact moment between the slider mass $M$ and the mobile nut from the stop unit.

Cylinder stroke s [mm]

225

Stepping motor characteristic

motor 3

\section{PK564-AE/PK564-BE}

Constant Current Driver Power Input: 24 VDC

Current: 0.75 A/Phase (4 Phases ON)

With Damper D6CL-8.0F : $\mathrm{JL}=140 \times 10^{-7} \mathrm{~kg} \cdot \mathrm{m}^{2}$

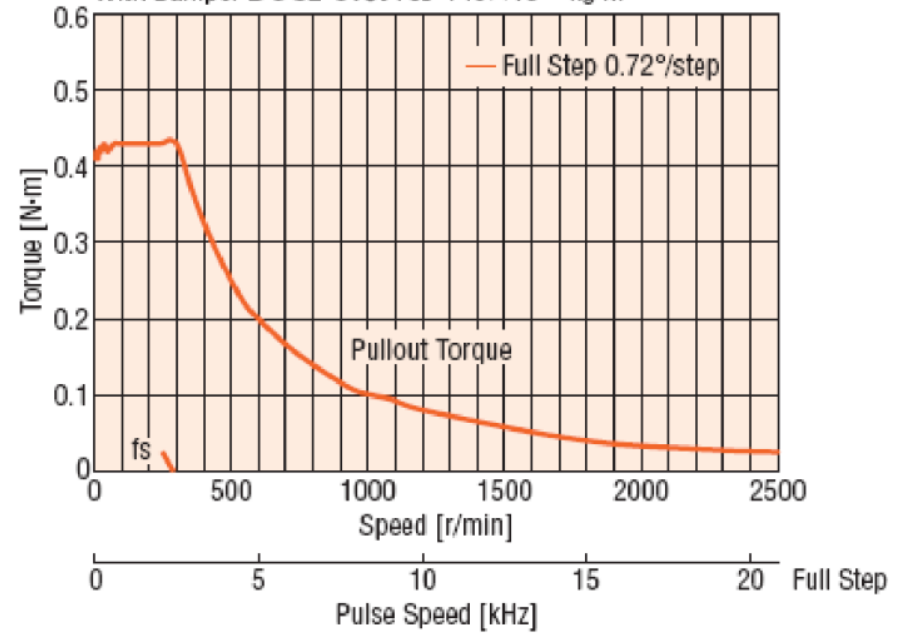

Stepsirevolution $\mathbf{N}$

Stepping motor stroke $x[\mathrm{~mm}]$

Fig. 2. Graphic user interface - main panel. 


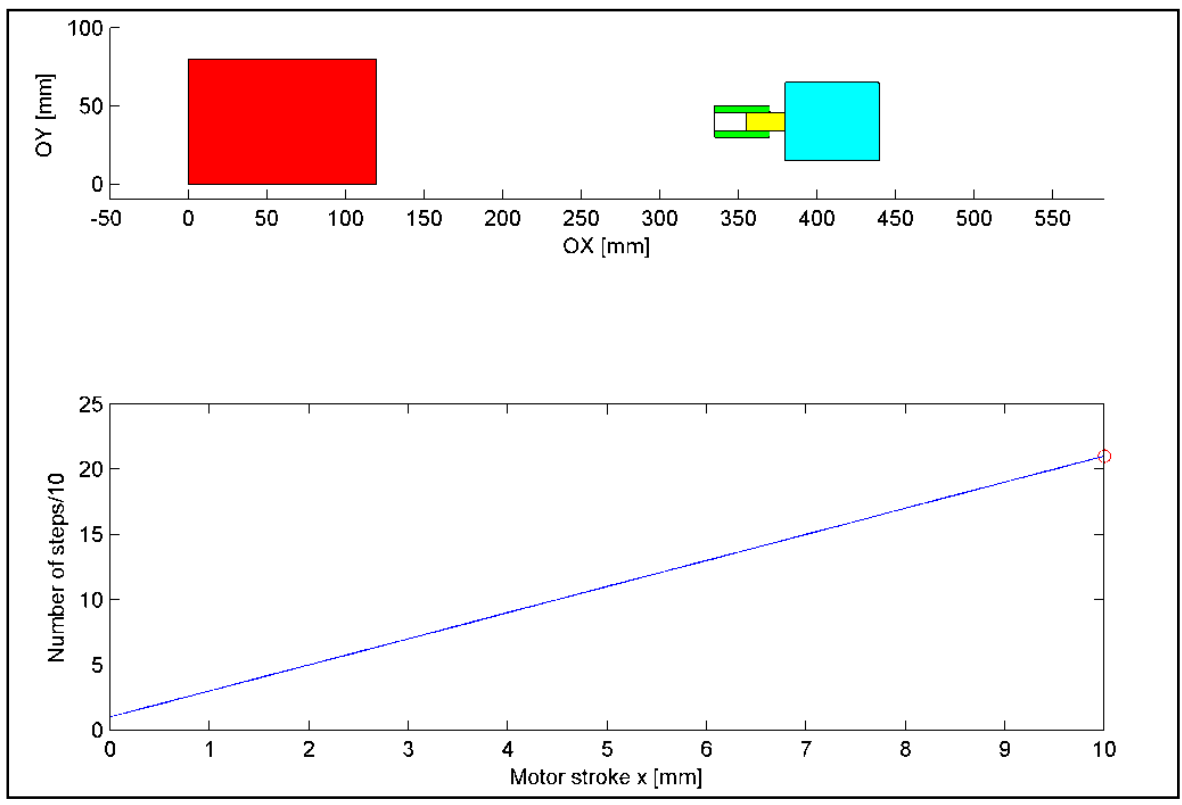

Fig. 3. Setting the stopper position with the stepping motor.

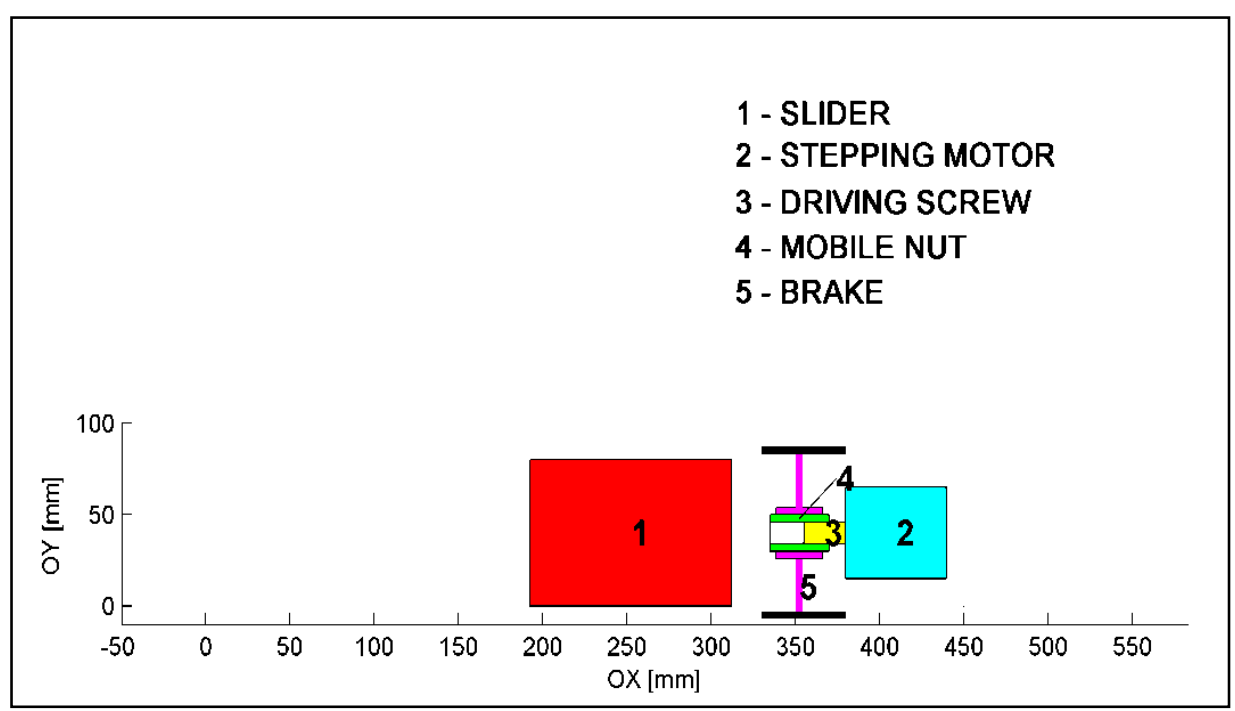

Fig. 4. The impact moment between the slider and the stopper unit.

The impact is shown in figure 4. Here the slider mass moves with the cylinder stroke. The simulation allows to compute stepping motors actuation times.

\section{Conclusions}

The simulation allows to compare different actuation solutions considering various stepping motors and actuation modes. A simulation allows for investigation of phenomena not directly measurable with the experimental apparatus. This research demonstrates the 
capabilities and limitations of advanced control strategies with stepping motors. Based on this simulation the authors developed an experimental setup.

\section{References}

1. Patent: US 6,802,242 B1, Pneumatic Circuit Control System (2004)

2. Patent US 7,021,191 B2, Accurate Fluid Operated Cylinder Positioning System (2006)

3. Gh. I. Gheorghe, I. Ilie, Optics \& Mechatronics, 46, 40 (2014)

4. Gh. Prisacaru, V. Carlescu, D. Olaru, Actuatori neconventionali în mecatronică (Ed. Tehnopres, 2013)

5. G. Belforte, $6^{\text {th }}$ trien. Int. Symp. on fluid control measurement and visualization: FLUCOME (2000)

6. C. Ferraresi, G. Quaglia, Proceedings of the Forth JHPS International Symposium on Fluid Power, Tokyo, 207 (1999)

7. X. Luo, H. Sun, J. Wang, American Control Conference on O'Farrell Street, San Francisco, CA, USA (2011)

8. Parker Hannifin Corporation Parker - Origa Glendale Heights, Illinois www.parker.com/pneu/rodless ORIENTAL MOTOR (EUROPA) GmbH www.orientalmotor.de 